\title{
NP-FARM: Negative and Positive Fuzzy Association Rule Mining in Transaction Dataset
}

\author{
Chandrasekar Ravi", Neelu Khare and N. Deepa \\ School of Information Technology and Engineering, VIT University, Vellore - 632014, Tamil Nadu, India; \\ chandrasekar.r@vit.ac.in
}

\begin{abstract}
Association Rule Mining (ARM) is a well recognized and interesting area of research in the field of Data Mining. In ARM, significant amount of research work has been reported. On the contrary, very less work has been reported for Negative Association Rule Mining (NARM). ARM concentrates only on positive rules while NARM explores negative rules. Also, most researchers have worked with discrete transaction dataset. So, in this paper, we propose a technique called Negative and Positive Fuzzy Association Rule Mining (NP-FARM), which mines both negative and positive association rules from a fuzzy transaction dataset. NP-FARM algorithm has been implemented and the experimental results determine the optimal minimum support threshold and optimal minimum confidence threshold for the given dataset. Also, the experimental results demonstrate that, as the size of the dataset increases, a negligible change in execution time is witnessed to mine the growing dataset.
\end{abstract}

Keywords: Association Rule Mining. Fuzzy Transactions, Positive and Negative Item Sets

\section{Introduction}

Data mining is defined as the process of extracting useful information from raw data by applying some of the wide range of data mining techniques which includes classification, clustering, etc... ${ }^{1-2}$ Frequent item set mining is one of the most popular technique used to extract useful patterns from the given data which could be transactional, relational, spatial, temporal, spatio-temporal, time-series, etc.., Frequent item sets are those which appear most frequently in the data using which association rules can be derived. Mathematically, frequent item sets could be considered as item sets whose support is greater than the minimum support threshold. Support indicates the frequency of occurrence of an item set in the given data. The association rule derived from the frequent item sets are considered to be strong if the confidence of the rule is greater than the minimum confidence threshold. Confidence is the conditional probability that the consequent occurs in the data set given that the antecedent occurred. Frequent item set mining, in general, explores only positive rules of the form $A=>B$, where $A \cap B=\{\}$ and item sets A, B are frequent item sets. Several algorithms exist for mining this type of rules ${ }^{3-4}$. Other forms of the association rules include $-\mathrm{A}=>\mathrm{B}, \mathrm{A}=>-\mathrm{B}$ and $\ulcorner\mathrm{A}=>-$ B. Very limited research has been done for exploring this type of rules. So, in this paper, an algorithm (NP-FARM) is proposed, which explores both negative and positive rules from a Fuzzy transaction dataset.

\section{Related Work}

Anis. ${ }^{5}$, provides an enhancement of Apriori algorithm for mining negative association rules from frequent absence and presence item set. The algorithm considers a discrete transaction data set. The algorithm takes the discrete transaction dataset as input and produces negative and positive frequent item set from which negative association rules are formed. The proposed algorithm is a modification of Apriori algorithm to derive negative rules based on frequent absence and presence item set. Jianchao. ${ }^{6}$ proposed an algorithm that mines both positive and negative frequent item sets and discovers negative and positive association rules. A fuzzy transaction dataset

${ }^{*}$ Author for correspondence 
was used. The support and confidence formulae were presented. The support and confidence were calculated based on the mean of product of the membership values of the items in the item set whose support was calculated. Wu. ${ }^{7}$ presented a model which extracts negative, positive association rules. The negative association between items sets is indicated by these negative item sets. A pruning method or correlation measure could increase the scalability. Antonie. ${ }^{8}$ proposed a technique which is an extension of the support confidence framework. Yuan. ${ }^{9}$ proposed a taxonomy based model for mining association rules and Hussain. ${ }^{10}$ presented a method based on entropy. Most of the above methods used discrete transaction dataset while the NP-FARM uses a Fuzzy transaction dataset and mines both negative and positive association rules.

\subsection{NP-FARM Description}

The Negative and Positive Fuzzy Association Rule Mining (NP-FARM) algorithms takes the Fuzzy transaction dataset, minimum support threshold and minimum confidence threshold as inputs. The algorithm consists of 2 phases. In the first phase, the negative and positive frequent item sets are generated using the Fuzzy transaction dataset and minimum support threshold. This is represented by Procedure Find_Negative_and_Positive_ Fuzzy_Frequent_Itemsets (dataset, minimum support). The support of an item set is defined as follows based on the property of Fuzzy that:

$$
\mu\left[{ }_{I 1 \text { AND I2 }}\right](t)=\operatorname{Minimum}\left[\mu_{I 1}(t), \mu_{I 2}(t)\right]
$$

Where,

$$
\mu[\mathrm{I}(\mathrm{t})] \text { - membership value of item set I in transaction } \mathrm{t}
$$

If the item set contains only positive items, then Support (S) of item set I is,

$$
\mathrm{S}(\mathrm{I})=\left[\sum_{\mathrm{t} \in \mathrm{D}} \operatorname{MIN}\left\{\mu\left[\mathrm{I}_{1}(\mathrm{t})\right], \mu\left[\mathrm{I}_{2}(\mathrm{t})\right], \ldots, \mu\left[\mathrm{I}_{\mathrm{k}}(\mathrm{t})\right]\right\}\right] /|\mathrm{D}|
$$

If the item set contains negative items, then Support (S) of item set I is defined as below,

for $I_{1}$ to $I_{k}$ in $\{c\}$, if $I_{k}$ is a negative item then, $\mu\left[I_{k}(t)\right]=$ $1-\mu\left[I_{k}(t)\right]$

$$
\mathrm{S}(\mathrm{I})=\left[\sum_{\mathrm{t} \in \mathrm{D}} \operatorname{MIN}\left\{\mu\left[\mathrm{I}_{1}(\mathrm{t})\right], \mu\left[\mathrm{I}_{2}(\mathrm{t})\right], \ldots, \mu\left[\mathrm{I}_{\mathrm{k}}(\mathrm{t})\right]\right\}\right] /|\mathrm{D}|
$$

Where,

$\mathrm{t}$ - fuzzy transactions in dataset D.

$\mathrm{I}_{\mathrm{k}}-\mathrm{k}^{\text {th }}$ item in item set $\mathrm{I}$

$\mu\left[I_{k}(t)\right]$ - membership value of item $I_{k}$ in transaction $t$
The above calculations are done by procedure Find_Support (item set). All the positive and negative 1 -itemsets are generated and their support is calculated using the above formula. If the support of an item is greater than minimum support threshold, then it is added in the frequent 1 -item set list. These frequent 1 -item set are used as candidates for generating frequent 2-item set. All frequent 2-item sets are generated and their support values are calculated. If support of an item set is greater than minimum support threshold, then, it is considered as candidate for next level item set generation. This procedure Candidate_generation (candidate k-1 item set) is repeated until no further candidates are available. Thus all the positive and negative frequent item sets are generated. In the next phase, association rules are generated using the Procedure Main_NP_FARM (dataset, min_sup, min_conf). for each of the frequent item sets I, all the non-empty subsets $\mathrm{P}$ are formed. This leads to the rules of the form $\mathrm{P}=>\mathrm{I}-\mathrm{P}$. The confidence of a rule is defined as follows:

$$
\text { Confidence }(\mathrm{P}=>\mathrm{I}-\mathrm{P})=\operatorname{Support}(\mathrm{I}) / \operatorname{Support}(\mathrm{P})
$$

If the confidence of a rule is less than minimum threshold confidence, then that rule is ignored. Thus all the strong negative and positive fuzzy association rules are mined. The NP-FARM algorithm, discussed below, is based on the Apriori algorithm ${ }^{1}$ and the algorithm proposed in ${ }^{5}$.

\subsection{NP-FARM Algorithm}

Algorithm: Negative and Positive, Fuzzy Association Rule Mining.

Input:

$\mathrm{D}$, a database of fuzzy transactions.

min_sup, the minimum support count threshold. min_conf, the minimum confidence count threshold.

Output:

Negative and Positive, Fuzzy Association Rules. Method:

Procedure Main_NP_FARM(D, min_sup, min_ conf)

A = Find_Negative_and_Positive_Fuzzy_

Frequent_Itemsets(D, min_sup);

for each itemset $\mathrm{I} € \mathrm{~A}$

for each non-empty subset $\mathrm{P} € \mathrm{I}$

$\mathrm{P}=>\mathrm{I}-\mathrm{P}$; 
if (Find_Support(I)/Find_Support $(\mathrm{P}))>=$ min_conf

$$
\mathrm{R}=\mathrm{R} \mathrm{U} \mathrm{P}
$$

// $\mathrm{R}$, the set of all negative and positive fuzzy association rules

return $\mathrm{R}$

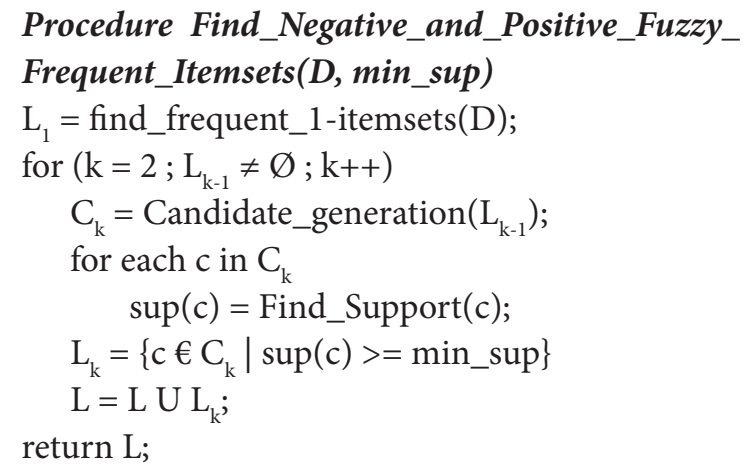

\section{procedure Find_Support(c)}

if all $\mathrm{k}$-items (i.e.) $\mathrm{I}_{1}$ to $\mathrm{I}_{\mathrm{k}}$ in $\{\mathrm{c}\}$ are positive then

$S(c)=\left[\sum_{t \in D} \operatorname{MIN}\left\{\mu\left[I_{1}(t)\right], \mu\left[I_{2}(t)\right], \ldots, \mu\left[I_{k}(t)\right]\right.\right.$ \}$] /|D|$

else

for $I_{1}$ to $I_{k}$ in $\{c\}$

if $I_{k}$ is a negative item then $\mu\left[I_{k}(t)\right]=1-$ $\mu\left[\mathrm{I}_{\mathrm{k}}(\mathrm{t})\right]$

$\mathrm{S}(\mathrm{c})=\left[\sum_{\mathrm{t} \in \mathrm{D}} \operatorname{MIN}\left\{\mu\left[\mathrm{I}_{1}(\mathrm{t})\right], \mu\left[\mathrm{I}_{2}(\mathrm{t})\right], \ldots\right.\right.$, $\left.\left.\mu\left[I_{k}(t)\right]\right\}\right] /|D|$

return $\mathrm{S}$;

procedure Candidate_generation $\left(L_{k-1}\right)$

for each itemset $l_{1} € \mathrm{~L}_{\mathrm{k}-1}$

for each itemset $\mathrm{l}_{2} \mathrm{v} \mathrm{L}_{\mathrm{k}-1}$

$$
\text { if }\left(1_{1}[1]=1_{2}[1]\right) \cap\left(1_{1}[2]=1_{2}[2]\right) \cap \ldots \cap
$$$$
\left(\mathrm{l}_{1}[\mathrm{k}-1]<\mathrm{l}_{2}[\mathrm{k}-1]\right) \text { then }
$$

$\mathrm{c}=1_{1}$ JOIN $1_{2}$;// join step: generate candidates

if has_infrequent_subset $\left(c, \mathrm{~L}_{\mathrm{k}-1}\right)$ then delete c; // prune step: remove unfruitful candidate

return $\mathrm{C}_{\mathrm{k}}$; else add $c$ to $\mathrm{C}_{\mathrm{k}}$;

procedure has_infrequent_subset $\left(c, L_{k-1}\right)$

for each $(\mathrm{k}-1)$-subset $\mathrm{s}$ of $\mathrm{c}$

if $s \notin \mathrm{L}_{\mathrm{k}-1}$ then

return TRUE;

return FALSE;

\section{Experimental Results}

The NP-FARM algorithm has been implemented using MATLAB $^{11}$. Fuzzy transaction data set containing 1000 transactions was given as input to NP-FARM. Table 1 shows the variation of the number of rules generated with the no. of input attributes for various min_sup and min_conf. Table 2 shows the variation of execution time (seconds) as the size of the dataset increases. Figure 1, 2, 3 shows the the variation of the number of rules generated with the no. of input attributes $(10,20,30,40,50)$ for various min_sup $(0.3,0.35,0.4)$ and min_conf. $(0.6,0.65$, 0.7 ). Based on the number of rules generated, the optimized min_sup is predicted to range between 0.3 and 0.35 and similarly optimized min_conf is predicted to range between 0.6 and 0.7. Figure 4 shows the variation of execution time (seconds) as the size of the dataset increases from 200 to 1000 records. The variations are observed to be negligible even though the size of the dataset is growing. Hence the execution time of NP-FARM algorithm is independent of the size of the dataset.

\begin{tabular}{|c|c|c|c|c|c|c|c|c|c|}
\hline \multirow{3}{*}{ No. of attr } & \multicolumn{9}{|c|}{ No. of NP-FARM Rcules } \\
\hline & \multicolumn{3}{|c|}{$\min \_$supp $=0.3$} & \multicolumn{3}{|c|}{ min_supp $=0.35$} & \multicolumn{3}{|c|}{$\min \_$supp $=0.4$} \\
\hline & $\begin{array}{c}\text { min_conf } \\
=0.6\end{array}$ & $\begin{array}{c}\text { min_conf } \\
=0.65\end{array}$ & $\begin{array}{c}\text { min_conf } \\
=0.7\end{array}$ & $\begin{array}{c}\min \_ \text {conf } \\
=0.6\end{array}$ & $\begin{array}{c}\text { min_conf } \\
=0.65\end{array}$ & $\begin{array}{c}\text { min_conf } \\
=0.7\end{array}$ & $\begin{array}{c}\text { min_conf } \\
=0.6\end{array}$ & $\begin{array}{c}\min \_ \text {conf } \\
=0.65\end{array}$ & $\begin{array}{c}\text { min_conf } \\
=0.7\end{array}$ \\
\hline 10 & 360 & 345 & 6 & 12 & 10 & 0 & 0 & 0 & 0 \\
\hline 20 & 1520 & 1406 & 0 & 20 & 20 & 0 & 0 & 0 & 0 \\
\hline 30 & 3480 & 3110 & 8 & 36 & 106 & 4 & 0 & 0 & 0 \\
\hline 40 & 6240 & 5830 & 34 & 68 & 44 & 7 & 0 & 0 & 0 \\
\hline 50 & 9800 & 9008 & 9500 & 164 & 296 & 3 & 0 & 0 & 0 \\
\hline
\end{tabular}

Table 1. Finding optimal support and confidence 
Table 2. Size of dataset vs time

\begin{tabular}{lccccc}
\hline \multirow{2}{*}{$\begin{array}{l}\text { No. of } \\
\text { Records }\end{array}$} & \multicolumn{4}{c}{ Execution Time (seconds) for min_sup $=\mathbf{0 . 3}$ and min_conf $=\mathbf{0 . 6}$} \\
\cline { 2 - 6 } & $\begin{array}{c}\text { No. of } \\
\text { attr = } \mathbf{1 0}\end{array}$ & $\begin{array}{c}\text { No. of } \\
\text { attr = 20 }\end{array}$ & $\begin{array}{c}\text { No. of } \\
\text { attr = 30 }\end{array}$ & $\begin{array}{c}\text { No. of } \\
\text { attr }=\mathbf{4 0}\end{array}$ & $\begin{array}{c}\text { No. of } \\
\text { attr = 50 }\end{array}$ \\
\hline 200 & 1.6 & 13.8 & 69.8 & 301.7 & 635.9 \\
400 & 1.7 & 14.0 & 69.9 & 301.9 & 636.2 \\
600 & 1.8 & 14.7 & 70.7 & 302.2 & 636.8 \\
800 & 1.8 & 15.2 & 70.4 & 302.7 & 637.2 \\
1000 & 1.9 & 15.7 & 71.7 & 303.1 & 637.5 \\
\hline
\end{tabular}

min_sup $=0.3$

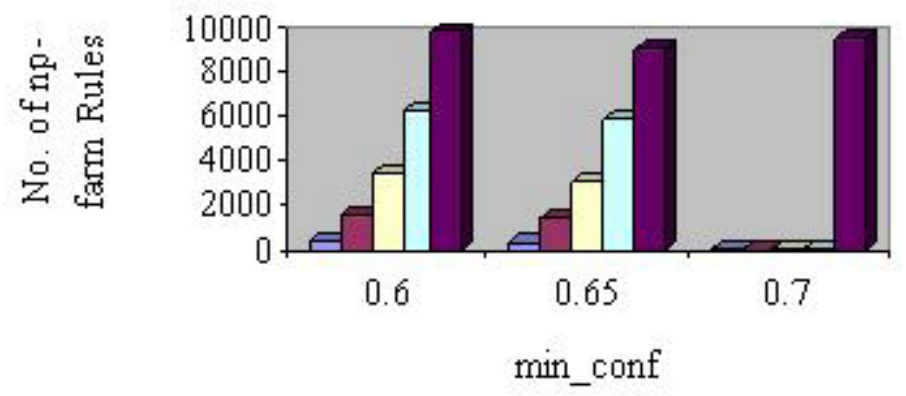

$\square 10$ attributes $\square 20$ attributes $\square 30$ attributes $\square 40$ attributes $\square 50$ attributes

Figure 1. Rules VS min_sup_and_conf

\section{min_sup $=0.35$}

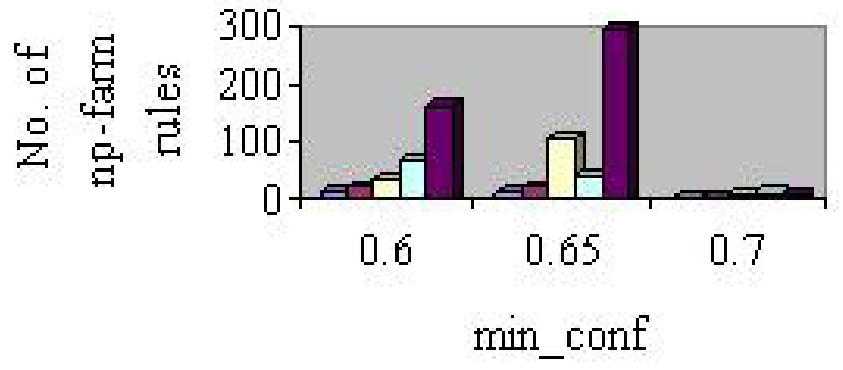

\section{$\square 10$ attributes $\square 20$ attributes $\square 30$ attributes 40 attributes 50 attributes}

Figure 2. Rules VS min_sup_and_conf 


$$
\text { min_sup }=0.4
$$

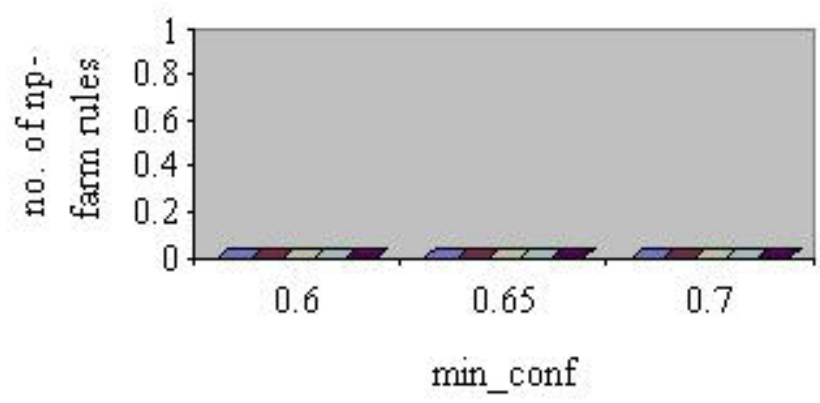

\section{0 attributes $\square 20$ attributes $\square 30$ attributes $\square 40$ attributes $\square 50$ attributes}

Figure 3. Rules VS min_sup_and_conf

$$
\text { min_sup }=0.3, \text { min_conf }=0.6
$$

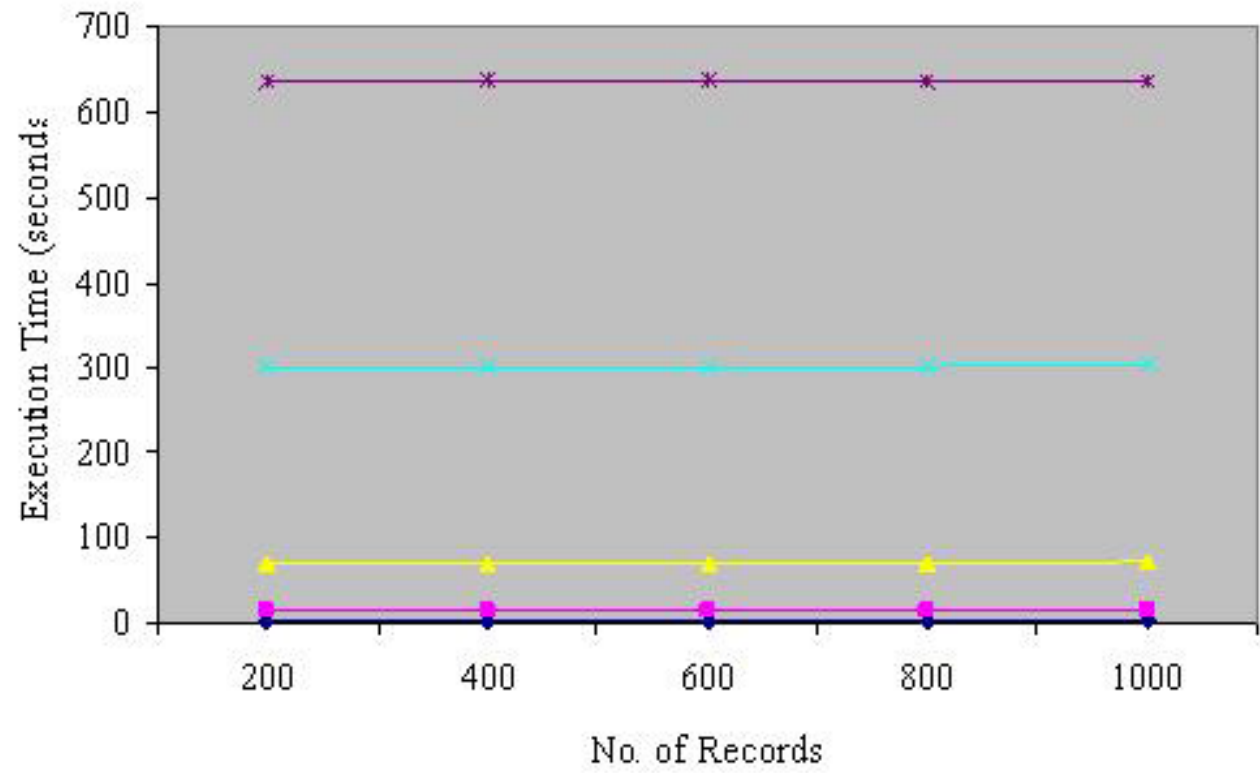

$$
\begin{aligned}
& \rightarrow-10 \text { attributes }--20 \text { attributes } \quad 30 \text { attributes } \\
& -40 \text { attributes } \rightarrow-50 \text { attributes }
\end{aligned}
$$

Figure 4. Size vs Execution Time

\section{Conclusion and Future Work}

In this paper, a technique has been proposed, called Negative and Positive Fuzzy Association Rule Mining (NP-FARM), which mines both negative and positive association rules from a fuzzy transaction dataset.
NP-FARM algorithm has been implemented and the experimental results determine the optimal minimum support threshold and minimum confidence threshold. Also, the experimental results demonstrate that the time required for mining the rules remains almost constant even if the size of the dataset increases. Hence the 
execution time of NP-FARM algorithm is independent of the size of the dataset. The derivation of membership function for calculating the membership values of the items in the transactions is not considered in this work. A Fuzzy dataset is just considered. In the future work, an appropriate membership function could be determined and some correlation measures could be included to prune the rules.

\section{References}

1. Han, J. \& Kamber, M. (2006). Data Mining Concepts and Techniques. San Francisco: Mogan Kaufmann Publishers.

2. Sumathi, S. \& Sivanandam, S. N. (2006). Introduction to Data Mining and its Applications. Berlin: Springer-Verlag.

3. Agrawal, R., Imielinski, T. \& Swami, A. (1993). Mining association rules between set of items in large database. Paper presented in ACM SIGMOD Conference.

4. Agrawal, R. \& Srikant, R. (1994). Fast Algorithms for Mining Association Rules. Paper presented in $20^{\text {th }}$ VLDB conference, Santiago.

5. Anis, S. A. K., Azuraliza, A. B. \& Abdul R. H. (2011, Nov. 22-24). Frequent Absence and Presence Itemset for
Negative Association Rule Mining. Paper presented at Intelligent Systems Design and Applications (ISDA), 2011 11th International Conference, Cordoba.

6. Jianchao, H, \& Mohsen, B. (2005, Dec. 21). Discovering Both Positive and Negative Fuzzy Association Rules in Large Transaction Databases. Journal of Advanced Computational Intelligence and Intelligent Informatics. Vol. 10 No. 3.

7. Wu, X., Zhang, C. \& Zhang, S. (2004). Efficient mining of both positive and negative association rules. ACM Transactions on Information Systems (TOIS) 22(3): 381405

8. Antonie, M. L. \& Zaïane, O. R. (2004). Mining positive and negative association rules: An approach for confined rules. Paper presented at Knowledge Discovery in Databases: PKDD.

9. Yuan, X., Buckles, B. P., Yuan, Z. \& Zhang, J. (2002). Mining negative association rules. Presented at Computers and Communications, ISCC, the Seventh International Symposium.

10. Hussain, F., Liu, H., Suzuki, E. \& Lu, H. (2000). Exception rule mining with a relative interestingness measure. Paper presented at Knowledge Discovery and Data Mining. Current Issues and New Applications.

11. http://www.mathworks.in/ 